The Astrophysical Journal, 523:L93-L96, 1999 September 20

(C) 1999. The American Astronomical Society. All rights reserved. Printed in U.S.A.

\title{
CORONAL HEATING BY MAGNETOHYDRODYNAMIC TURBULENCE DRIVEN BY REFLECTED LOW-FREQUENCY WAVES
}

\author{
W. H. Matthaeus, ${ }^{1}$ G. P. Zank, ${ }^{1}$ S. Oughton,${ }^{2}$ D. J. Mullan, ${ }^{1}$ and P. DmitruK ${ }^{1}$ \\ Received 1999 June 4; accepted 1999 July 16; published 1999 August 19
}

\begin{abstract}
A candidate mechanism for the heating of the solar corona in open field line regions is described. The interaction of Alfvén waves, generated in the photosphere or chromosphere, with their reflections and the subsequent driving of quasi-two-dimensional MHD turbulence is considered. A nonlinear cascade drives fluctuations toward short wavelengths which are transverse to the mean field, thereby heating at rates insensitive to restrictive Alfvén timescales. A phenomenology is presented, providing estimates of achievable heating efficiency that are most favorable.
\end{abstract}

Subject headings: MHD — Sun: corona — turbulence

\section{INTRODUCTION}

An acceptable model (Habbal et al. 1995; McKenzie, Banaszkiewicz, \& Axford 1995) for the origin of the high-latitude fast solar wind must produce significant heat deposition within the first several solar radii above the photosphere to account for the observed rapid acceleration of the wind (Grall et al. 1996). Theory and observations indicate that the magnetic field plays a crucial role in the heating process (Axford \& McKenzie 1997; Culhane 1998; Ulmschneider, Priest, \& Rosner 1991), with Alfvén waves and magnetic reconnection identified as probable agents. Here we present a mechanism for the heating of the coronal plasma, taking these features into account while avoiding the possible disadvantages of heating models that rely on high-frequency waves (Axford \& McKenzie 1997; McKenzie et al. 1995; Tu \& Marsch 1997).

The high-speed solar wind probably depends upon ion heating close to the Sun, since Alfvén wave and electron pressures are almost certainly inadequate. Emphasizing this point, McKenzie et al. (1995) and Habbal et al. (1995) postulate ion heating/dissipation described by the ad hoc functional form (Holzer \& Axford 1970) $Q \equiv Q_{0} \exp \left[-\left(r-r_{0}\right) / L\right]$, where $r$ is heliocentric distance, $r_{0}$ is a constant, and $L$ is a prescribed dissipation length. Remarkably, both models obtain good agreement with observations for $L \simeq 0.5-0.7 R_{s}$ and reasonable coronal base parameters, accounting for correct speed and particle flux, outer coronal densities, high inner coronal ion temperatures, and rapid acceleration of the wind associated with low coronal base densities. This general picture is strongly supported by recent results from SOHO (Kohl et al. 1997). However, a full physical understanding of the heating mechanism remains to be established.

A major challenge in describing coronal heating at the base of the high-latitude, fast wind is that these are open magnetic field line regions. Heating of closed coronal magnetic loops has been studied widely, particularly in connection with Parker's (1972) model of energy injection by photospheric footpoint motion and subsequent dissipation in current sheets. While discussion of the closed-loop heating process (van Ballegooijen 1986; Priest et al. 1998) spans several decades, there appears to be wide consensus that the energy originates in

\footnotetext{
${ }^{1}$ Bartol Research Institute, University of Delaware, Newark, DE 19716; yswhm@bartol.udel.edu.

${ }^{2}$ Department of Mathematics, University College London, London WC1E 6BT, England, UK.
}

photospheric motions and that magnetically dominated coronal activity eventually results in plasma heating. The problem in extending the closed loop models to open field line regions is clear-excitations supplied in the photosphere are expected to be transported outward rapidly. Informed estimates suggest Alfvén speed values of $V_{\mathrm{A}} \approx 200-2000 \mathrm{~km} \mathrm{~s}^{-1}$ in the crucial first few solar radii of the corona. Fluctuation energy may thus be lost too rapidly from the region $R<2 R_{s}$, unless there is some feature of the process that amplifies or enhances the heating process. Heating at larger $r$ cannot account for the recent $S O H O$ UVCS observations (e.g., Kohl et al. 1997) and also would not accelerate the fast wind in accord with remote sensing data (Grall et al. 1996).

The essence of the Axford \& McKenzie (1997) suggestion is that rapid heating can be accomplished by high-frequency Alfvén waves of network origin that experience rapid cyclotron damping in the lower corona. This model provides an ubiquitous source of wave energy and a straightforward explanation for several features of $\mathrm{SOHO}$ observations that appear to be signatures of cyclotron absorption mechanisms. However, large wave energy flux at high frequencies is required, up to perhaps the kilohertz range. This might be feasible if there is a large enhancement of $\mathrm{kHz}$ power at the coronal base. Alternatively, as noted by Tu \& Marsch (1997), a very broad band of $1 / f$ wave power extending to the requisite frequencies would suffice. Axford \& McKenzie (1997) offer plausibility arguments for the existence of a substantial wave population at the requisite small parallel length scales. However, lacking direct observational evidence for this, it seems reasonable to explore similar models that relax the problematic constraint of highfrequency waves. Here we describe the framework of a model that involves turbulent heating due to low-frequency magnetohydrodynamic (MHD) fluctuations.

\section{STRUCTURE OF THE MODEL}

Figure 1 illustrates the proposed mechanism. An influx of waves from the coronal base propagates along the background field $\boldsymbol{B}_{0}$, driving quasi-two-dimensional MHD turbulence and dissipation of energy at small perpendicular scales. Specifically, the continual energy supply for turbulent heating results from Alfvénic fluctuations, which are generated below the transition region, either in the chromosphere or the photosphere. Some of these waves propagate into the corona where they experience non-WKB reflection off the large-scale density and field gradients (Hollweg 1981, 1996; Velli 1993; Zhou \& Mat- 


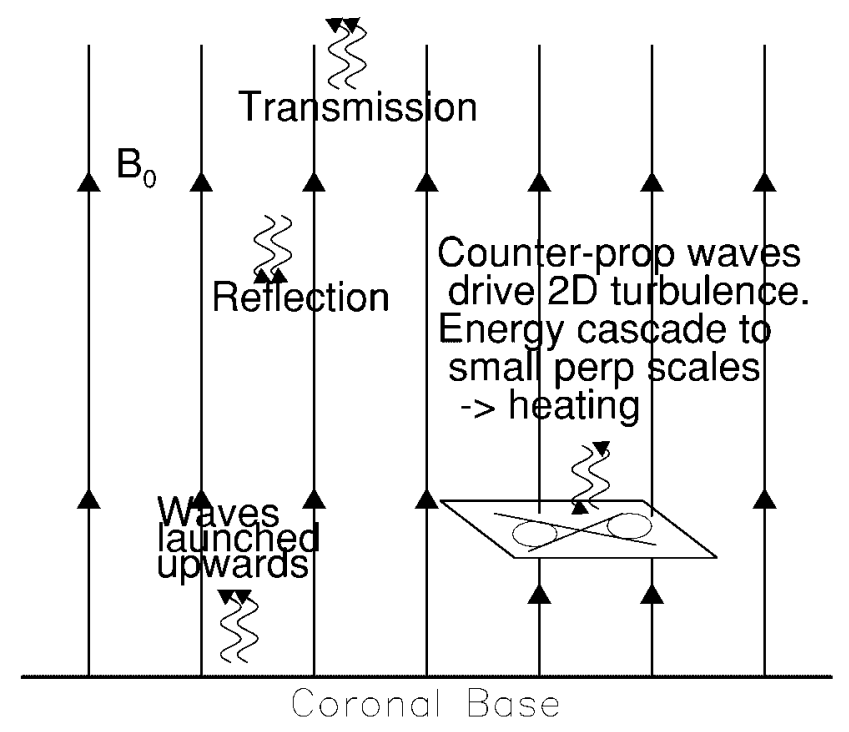

FIG. 1.-Cartoon sketch of the basic physics underlying the proposed coronal heating mechanism.

thaeus 1990). Reflection is required because (in the incompressible limit) there are no nonlinear couplings when fluctuations propagate in one direction only. When sufficient energy resides in low-frequency quasi-two-dimensional fluctuations, i.e., wavevectors almost perpendicular to the (average) coronal magnetic field $\boldsymbol{B}_{0}$, then the waves drive turbulence (Shebalin, Matthaeus, \& Montgomery 1983; Oughton, Ghosh, \& Matthaeus 1998). At large Reynolds numbers, quasi-twodimensional fluctuations engage in a turbulent cascade involving successive reconnection of poloidal flux structures (see e.g., Matthaeus \& Lamkin 1986), thereby transferring energy to smaller (perpendicular) scales where it is dissipated efficiently.

The population of MHD scale fluctuations can be thought of as two distinct types. First, there are low-frequency waves that can be grouped with the quasi-two-dimensional fluctuations because their wave period $\tau_{\mathrm{A}}$ is comparable to, or greater than, the characteristic nonlinear ("eddy turnover") timescale $\tau_{\mathrm{NL}} \sim \lambda_{\perp} / Z$ of the quasi-two-dimensional fluctuations $\left(\lambda_{\perp}\right.$ is a characteristic energy-containing length scale of the dynamical structures transverse to the mean magnetic field $B_{0} ; Z^{2}$ is a measure of the quasi-two-dimensional turbulence energy - see below). By contrast, a second population of higher frequency waves have $\tau_{\mathrm{A}}<\tau_{\mathrm{NL}}$. Their wave periods are short compared to the nonlinear time, but they need not have frequencies as high as the proton gyrofrequency. Let us now nominally distinguish between these regimes for coronal parameters. First, we estimate $\lambda_{\perp}$ to be no more than the network spacing of $30,000 \mathrm{~km}$ (Axford \& McKenzie 1997) and perhaps an order of magnitude less. Based upon the recent analysis of Chae, Schühle, \& Lemaire (1998), we take $Z \approx 30 \mathrm{~km} \mathrm{~s}^{-1}$. Thus, the nonlinear time may be in the range of $100-1000 \mathrm{~s}$, and coronal waves with periods of several hundred seconds and longer may efficiently drive quasi-two-dimensional turbulence.

The decomposition of MHD fluctuations into high- and lowfrequency components (Oughton et al. 1998; Kinney \& McWilliams 1998) is essential to understanding the MHD cascade process. When there is a very strong $B_{0}$, the propagation effects strongly distinguish the two populations, and the lowfrequency dynamics are referred to as reduced MHD or "RMHD" (Montgomery 1982; Zank \& Matthaeus 1992). The low-frequency excitations engage in vigorous nonlinear cou- plings (Hossain et al. 1995), producing turbulence and robust heating at a rate of order $\sim Z^{3} / \lambda_{\perp}$ without regard for the value of $B_{0}$. This description of quasi-two-dimensional turbulent decay is analogous to the Taylor-Karman-Howarth phenomenology (e.g., von Kármán \& Howarth 1938) for hydrodynamic turbulence, appropriately modified for anisotropic low beta (plasma pressure/magnetic pressure) MHD turbulence.

Low-frequency modes are also distinctive in that they can undergo non-WKB "mixing" reflections (Zhou \& Matthaeus 1990) off gradients in the mean fields. The presence of large gradients in the lower corona indicates that significant nonWKB reflection is likely. Observations suggest a significant population of radially elongated structures in the corona (Grall et al. 1997). Moreover, both theory (Zank \& Matthaeus 1992, 1993) and simulations (Shebalin et al. 1983; Oughton et al. 1998) favor the development of states dominated by quasi-twodimensional fluctuations/turbulence for strong $B_{0}$ and plasma beta $\ll 1$ as is expected in the corona.

By contrast, for damping of high-frequency, parallel-propagating waves, the cascade in the parallel wavenumber direction is exceedingly slow (Tu \& Marsch 1997) and direct cyclotron resonance absorption occurs for progressively lower frequencies since the cyclotron frequency decreases with increasing altitude. High-frequency modes experience only weak nonlinear couplings and weak WKB reflections and would thus be transported rapidly through the corona, subject only to direct kinetic damping (Axford \& McKenzie 1997; McKenzie et al. 1995). Although the origin of the influx of waves may be essentially the same for both types of models, we do not suffer from the restriction of requiring the power in high frequencies, due to the rapid transverse turbulent cascade. In fact, because reflection is expected to be more efficient at lower frequencies, turbulent damping should actually work better for lowfrequency wave input (Moore et al. 1991). This may be an advantage if low-frequency fluctuations are favored in the generation mechanism, as we suspect is the case. Moreover, insensitivity of quasi-two-dimensional decay rates to $B_{0}$ may help explain the relative constancy of quantities like the solar wind mass flux and the coronal temperature.

\section{MHD TURBULENCE PHENOMENOLOGY}

A phenomenological approach is useful to demonstrate the essential physics of the ideas described above. Consider a typical sample of the relevant parts of the corona, as suggested by Figure 1. Rather than solve a detailed model that includes wave propagation, turbulence, coronal structure, and boundary conditions, we employ a simple MHD model averaged over this "box."

We follow the quasi-two-dimensional fluctuation energy using the Elsässer energies $Z_{ \pm}^{2} \equiv\left\langle\left|z_{ \pm}\right|^{2}\right\rangle$; the usual Elsässer variables are related to the (locally incompressible) plasma velocity fluctuation $\boldsymbol{v}$ and magnetic fluctuation $\boldsymbol{b}$ as $z_{ \pm}=\boldsymbol{v} \pm$ $\boldsymbol{b} /(4 \pi \rho)^{1 / 2}$, with $\rho$ the local mean mass density. Energy in fluctuations having cross helicity corresponding to upward propagation is denoted by $Z_{-}^{2}$. Correspondingly, $Z_{+}^{2}$ is the "downward" energy. The total turbulence energy (up to a numerical factor) is $Z^{2}=Z_{-}^{2}+Z_{+}^{2}$. Low-frequency upward-propagating waves continually enter the box from below, supplying fluctuation energy (per unit volume) at a rate $F$. Meanwhile, energy is lost to the reservoir of upward energy due to wave propagation through the top of the box at the Alfven speed $V_{\mathrm{A}}$. Reflections within the box involve conservative exchanges of energy between $Z_{+}^{2}$ and $Z_{-}^{2}$.

A particularly simple phenomenology can be written for tur- 


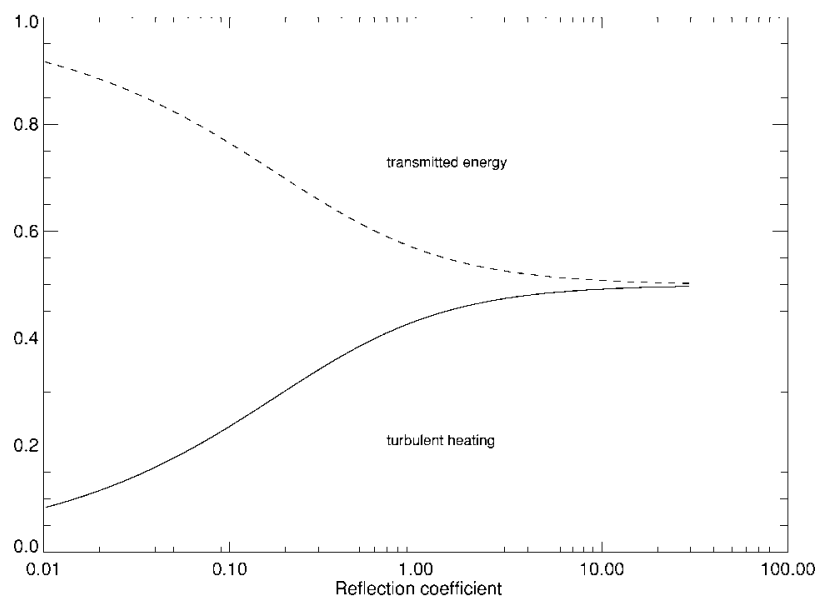

FIG. 2.-Steady rates of energy lost through transmission (dotted line) and through turbulent heating (solid line) for the model with supplied energy flux $F=1$ and transmission rate $T=1$, for varying reflection coefficients $R$.

bulent spectral transfer and associated heating of quasi-twodimensional MHD in a moderate or very strong mean magnetic field (Hossain et al. 1995). Incorporating energy supply, reflection, and transmission, we introduce a volume-averaged three-equation model:

$$
\begin{aligned}
& \frac{d Z_{-}^{2}}{d t}=-\alpha \frac{Z_{-}^{2} Z_{+}}{\lambda_{\perp}}+F-R^{-} Z_{-}^{2}+R^{+} Z_{+}^{2}-T Z_{-}^{2}, \\
& \frac{d Z_{+}^{2}}{d t}=-\alpha \frac{Z_{+}^{2} Z_{-}}{\lambda_{\perp}}+R^{-} Z_{-}^{2}-R^{+} Z_{+}^{2}, \\
& \frac{d \lambda_{\perp}}{d t}=-\beta \frac{\lambda_{\perp}}{Z^{2}}\left[\frac{d Z^{2}}{d t}\right] .
\end{aligned}
$$

In these equations, $\lambda_{\perp}$ is a single similarity scale describing energy decay for both upward- and downward-type fluctuations. The MHD Karman-Taylor constants are chosen as $\alpha=$ 1 and $\beta=1 / 2$, corresponding to constant turbulent Reynolds number (Matthaeus, Zank, \& Oughton 1996). A related model was used recently to describe solar wind heating in the outer heliosphere (Matthaeus et al. 1999). Transmission from the top of the box is at a volumetric rate $T Z_{-}^{2}$, where the transmission rate is $T \sim V_{\mathrm{A}} / L$ for box size $L$ in the vertical direction. Reflection rates $R^{-}$and $R^{+}$provide for internal reflections of both upward and downward fluctuations and will depend upon gradients of the large-scale flow, magnetic field, and density. Presently we shall consider only the special case $R^{+}=R^{-} \equiv R$. For low-frequency waves in the lower corona and low wind speed $U \ll V_{\mathrm{A}}$, we expect $R \sim V_{\mathrm{A}} / \Delta$, where $\Delta$ is the typical scale height for radial changes in the Alfvén speed (Hollweg 1981, 1996; Velli 1993; Zhou \& Matthaeus 1990).

\section{SOLUTIONS}

In seeking relevant solutions to equations (1)-(3), we solve the initial value problem numerically, obtaining a stable steady state after approximately 50 eddy turnover times. We adopt normalized units corresponding to the initial turbulence length and timescales, $\lambda_{0}$ and $\tau_{0}=\lambda_{0} / Z_{0}$, respectively. For the low beta corona, we expect RMHD orderings. To allow for low frequencies, the vertical box size must be large compared to the transverse similarity scale $L \gg \lambda_{\perp}$ (see, e.g., van Balle-

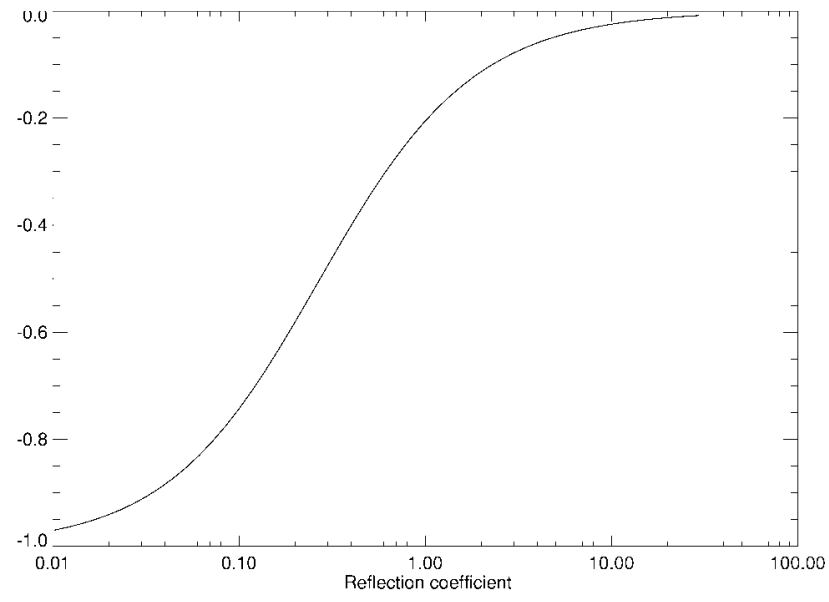

FIG. 3.-Steady values of normalized cross helicity $\sigma_{c}$ for varying reflection strength $R$. The other parameters are as in Fig. 2: $(F=1, T=1), \alpha=1, \beta=$ $1 / 2$. Note that $\sigma_{c}=-1$ corresponds to the absence of downward modes.

gooijen 1986). With $Z_{0}=1$ and $\lambda_{0}=1$, strong driving corresponds to $F=1$. In view of the condition on quasi-twodimensional turbulence timescales, $Z / \lambda_{\perp} \gtrsim V_{\mathrm{A}} / \lambda_{\|}$(Montgomery 1982; Zank \& Matthaeus 1992), where $\lambda_{\|}$is the driven parallel wavelength, we require that $\lambda_{\|}<L$. Thus, relevant values of the transmission rate satisfy $T \sim V_{A} / L \leq 1$. On the other hand, reflection rates may be either weak or strong, and thus both $R>1$ regimes and $R<1$ regimes are physically reasonable.

Figure 2 illustrates results for a varying reflection rate $R$, holding the transmission rate constant at its highest relevant value $T=1$. Shown are steady values of the transmitted energy per unit time, $T Z_{-}^{2}$, and the corresponding steady turbulent heating rate

$$
\epsilon=\alpha \frac{Z_{-}^{2} Z_{+}+Z_{+}^{2} Z_{-}}{\lambda_{\perp}}=F-T Z_{-}^{2} .
$$

For strong reflection $(R \gg 1)$, nearly half the wave energy flux is processed by the turbulence and deposited as heat within the box. However, it is also evident that one requires only modest values of $R(\geq 0.02)$ to cause at least $10 \%$ of the wave energy to be deposited as heat. By contrast, in the absence of turbulence, energy may be stored in the coronal box by reflections (see Hollweg 1981, 1996), but in a steady state all the energy is transmitted. One can also easily characterize the steady transmitted energy flux $T Z_{-}^{2}$ for the case of very strong reflection. One finds asymptotically for $R \gg 1$ (and for $\alpha=1$ and $\beta=$ $1 / 2$ ) that

$$
T Z_{-}^{2}=F-\epsilon \rightarrow \frac{T \sqrt{T^{2}+8 F}-T^{2}}{4},
$$

which verifies that $\epsilon=F / 2$ for $T=1$ as $R \rightarrow \infty$, as seen in Figure 2.

An interesting feature of the present model is that in steady state there is a mixture of upward- and downward-propagating fluctuations. The normalized cross helicity $\sigma_{c}=\left(Z_{+}^{2}-\right.$ $\left.Z_{-}^{2}\right) /\left(Z_{+}^{2}+Z_{-}^{2}\right)$ provides a measure of the dominance of either upward- or downward-type fluctuations. Figure 3 shows the steady state values of $\sigma_{c}$ for $F=1$ and $T=1$ and varying $R$. For all but the smallest values of $R$ there is a nonnegligible level of downward-propagating fluctuations. In contrast, the Axford-McKenzie direct cyclotron damping model makes no 
clear statement about the level of downward-traveling waves and contains no mechanism for producing them. Here, for even moderate reflection $R \geq 1$, the steady levels of $Z_{+}^{2}$ and $Z_{-}^{2}$ become nearly equal, as there is no net cross helicity as $R \rightarrow \infty$. By inferring values of $\sigma_{c}$, remote sensing diagnostics may be able to provide an observational distinction between the present model and the Axford-McKenzie model. Upward wave fluxes are detected in the chromosphere (Ulrich 1996). Detection of wave fluxes in the transition region (Doyle et al. 1998) is less definitive at present. As for the corona, Chae et al. (1998) argue that upward waves are not present, and they prefer to interpret their results in terms of small scale MHD turbulence. It is not clear to us if these observations are conclusive or if they might be consistent with a mixture of turbulent $Z_{-}$and $Z_{+}$fluctuations such as we foresee in the present model.

\section{DISCUSSION}

We have described a novel mechanism for heating open field line regions of the corona. The basic features are the generation of an upward-propagating low-frequency Alfvén wave flux at the coronal base, the occurrence of non-WKB reflection due to gradients in the Alfvén speed, and the subsequent driving of quasi-two-dimensional MHD turbulence through the interaction of counterpropagating fluctuations. We have examined the feasibility of the above mechanism through a simple phenomenological treatment of energy supply, reflection, turbulent decay, and transmission. The reflection rate $R$ has a significant influence on the heating efficiency of this process, and, for reasonable values of $R \geq 1$, one finds that nearly half the input wave energy is deposited as heat in the box representing the lower corona.
This preliminary result encourages further investigation along these lines. One would like to see whether high efficiencies are obtained in more complete models incorporating detailed treatments of reflection and transmission (Hollweg 1981, 1996; Velli 1993) and more realistic chromospheric and coronal fields (Gabriel 1976; Axford \& McKenzie 1997). The turbulence itself can also be treated more explicitly through direct MHD simulation. Another useful approach may be development of a phenomenological transport theory for the lower corona, building on similar theories applied to the solar wind (Zhou \& Matthaeus 1990; Tu \& Marsch 1993; Matthaeus et al. 1999).

We have suggested that anisotropic spectral transfer can provide an efficient heating mechanism in open field line coronal regions. This conclusion depends upon reflection which produces a crucial flux of counterpropagating waves. The heating requires that the quasi-two-dimensional turbulence level become self-sustaining (as it does in the present model). It thus appears that heating of open field line regions is more like closed field line heating than has been thought previously, counterpropagating waves and small transverse scales being important in both cases. One would also like to understand eventually how kinetic processes damp the small-scale excitations that are spectrally cascaded to oblique wavevectors. Possible mechanisms include ion-cyclotron and Landau damping (Leamon et al. 1998) as well as nonlinear processes such as beam instabilities or mode conversion and damping.

This work is supported by NASA (grants NAG5-7164, NAG5-6570, and NAG5-8134) and the UK PPARC (GR/ K98711).

\section{REFERENCES}

Axford, W. I., \& McKenzie, J. F. 1997, in Cosmic Winds and the Heliosphere, ed. R. Jokippi, C. P. Sonnet, \& M. S. Giampapa (Arizona: Univ. Arizona Press), 31

Chae, J., Schühle, U., \& Lemaire, P. 1998, ApJ, 505, 957

Culhane, J. L. 1997, Adv. Space Res., 19, 1839

Doyle, J. G., van den Oord, G. H. J., O’Shea, E., \& Banerjee, D. 1998, Sol. Phys., 181, 51

Gabriel, A. H. 1976, Philos. Trans. R. Soc. London A, 281, 339

Grall, R. R., Coles, W. A., Klinglesmith, M. T., Breen, A. R., Williams, P., Markkanen, J., \& Esser, R. 1996, Nature, 379, 429

Grall, R. R., Coles, W. A., Spangler, S. R., Sakurai, T., \& Harmon, J. K. 1997, J. Geophys. Res., 102, 263

Habbal, S., Esser, R., Guhathakurta, M., \& Fisher, R. 1995, Geophys. Res. Lett., 22, 1465

Hollweg, J. V. 1981, Sol. Phys., 70, 25

- 1996, in AIP Conf. Proc. 382, Solar Wind Eight, ed. D. Winterhalter, J. T. Gosling, S. R. Habbal, W. S. Kurth, \& M. Neugebauer (New York: AIP), 327

Holzer, T. E., \& Axford, W. I. 1970, ARA\&A, 8, 31

Hossain, M., Gray, P. C. Pontius, D. H., Jr., Matthaeus, W. H., \& Oughton, S. 1995, Phys. Fluids, 7, 2886

Kinney, R., \& McWilliams, J. C. 1998, Phys. Rev. E, 57, 7111

Kohl, J. L., et al. 1997, Sol. Phys., 175, 613

Leamon, R. J., Matthaeus, W. H., Smith, C. W., \& Wong, H. K. 1998, ApJ, $507, \mathrm{~L} 181$
Matthaeus, W. H., \& Lamkin, S. L. 1986, Phys. Fluids, 29, 2513

Matthaeus, W. H., Zank, G. P., \& Oughton, S. 1996, J. Plasma Phys., 56, 659

Matthaeus, W. H., Zank, G. P., Smith, C. W., \& Oughton, S. 1999, Phys. Rev. Lett., 82, 3444

McKenzie, J., Banaszkiewicz, M., \& Axford, W. I. 1995, A\&A, 303, L45

Montgomery, D. C. 1982, Phys. Scr., T2/1, 83

Moore, R. L., Musielak, Z. E., Suess, S. T., \& An, C.-H. 1991, ApJ, 378, 347

Oughton, S., Ghosh, S., \& Matthaeus, W. H. 1998, Phys. Plasmas, 5, 4235

Parker, E. N. 1972, ApJ, 174, 499

Priest, E. R., Foley, C. R., Heyvaerts, J., Arber, T. D., Culhane, J. L., \& Acton, L. W. 1998, Nature, 393, 545

Shebalin, J. V., Matthaeus, W. H., \& Montgomery, D. 1983, J. Plasma Phys., 29, 525

Tu, C.-Y., \& Marsch, E. 1993, J. Geophys. Res., 98, 1257 1997, Sol. Phys., 171, 363

Ulmschneider, P., Priest, E. R., \& Rosner, R. 1991, Mechanisms of Chromospheric and Coronal Heating (Berlin: Springer)

Ulrich, R. K. 1996, ApJ, 465, 436

van Ballegooijen, A. A. 1986, ApJ, 311, 1001

Velli, M. 1993, A\&A, 270, 304

von Kármán, T., \& Howarth, L. 1938, Proc. R. Soc. London A, 164, 192

Zank, G. P., \& Matthaeus, W. H. 1992, J. Plasma Phys., 48, 85 1993, Phys. Fluids A, 5, 257

Zhou, Y., \& Matthaeus, W. H. 1990, J. Geophys. Res., 95, 10291 